\title{
Oxidative stress and the evolutionary origins of preeclampsia
}

\author{
Michael G Elliot \\ St. John's College, Cambridge, CB2 1TP, United Kingdom \\ Email:me378@cam.ac.uk
}

\begin{abstract}
In this speculative paper, I consider the relationship between oxidative stress and the evolution of placentation in eutherian mammals. I argue that epitheliochorial placentation, in which fetal tissues remain separated from maternal blood throughout gestation, has evolved as a protective mechanism against oxidative stress arising from pregnancy, particularly in species with unusually long gestation lengths and unusually large placentas. Human beings comprise an unusual species which has the life history characteristics of an epitheliochorial species but exhibits hemochorial placentation, in which fetal tissues come into direct contact with maternal blood. I argue that the risk of preeclampsia has arisen as a consequence of the failure of human beings to evolve epitheliochorial placentation.
\end{abstract}

Keywords: Preeclampsia, Hemochorial placentation, Epitheliochorial placentation, Oxidative stress

\section{Introduction}

In many mammalian species, pregnancy involves deep invasion by placental tissues - which are of fetal origin - into the wall of the uterus, generating intimate and complex interactions between the physiologies of mother and offspring during gestation. Human beings have an invasive form of placentation in which fetal cells invade into the inner third of the myometrium and take control of the local maternal circulatory system by transforming her uterine spiral arteries into inelastic tubes no longer subject to maternal vasodilatory control (Lyall, 2005). The apparent necessity of this relatively deep invasion of the uterine wall during human gestation has been held to 
predispose human beings to diseases related to inadequate (and excessive) invasion (Crosley et al., 2013; Elliot and Crespi, 2015; Pijnenborg et al., 2008; Rosenberg and Trevathan, 2007; Robillard et al., 2003), most prominently preeclampsia (in which inadequate invasion and a consequent failure to remodel the maternal vasculature is a universal feature). Hence, the existence of preeclampsia in human beings represents an evolutionary conundrum given what must be a vast reproductive cost of preeclampsia to human populations over historical times, how has the condition been retained by natural selection? What benefits of deeply invasive placentation could outweigh such costs?

Invasive placentation, in the sense described here, is hemochorial placentation. In species with hemochorial placentas (including monkeys, apes, rodents, rabbits, many bats and insectivores, and several African shrew-like mammals) the fetal placental tissues breach maternal blood vessels, causing maternal blood to wash directly over the surface of the placenta and through its complex sinuses before being returned to uterine veins. Direct contact between circulating maternal blood and fetal tissues (which bear antigens of paternal and hence foreign origin) gives rise to a range of complex immunological and physiological complications, likely including preeclampsia. Yet, many mammalian species exhibit quite different forms of placentation which appear to evade such complications entirely. Species with epitheliochorial placentas (including ruminants, whales, and the lower primates) have the least invasive form. The placenta not only fails to breach maternal blood vessels, but it never even breaches the epithelial tissues lining the wall of the uterus. Maternal blood in such species is generally bound with maternal blood vessels, and fetal tissues never come into contact with it. Species with endotheliochorial placentas (including almost all carnivores, along with some bats, South American mammals and some African mammals) lie somewhere between these two extremes: the placenta invades past the maternal epithelium but does not invade maternal blood vessels, such that maternal blood and fetal tissues are always separated by the endothelium of maternal capillary walls. It is noteworthy that neither preeclampsia, nor any condition very similar to it, has been observed in a species with epitheliochorial or endotheliochorial placentation.

Historically, epitheliochorial placentation has been regarded as a "primitive" condition from which more advanced mammalian species with deeply invasive placentation have arisen. However, the expansion of studies of comparative placentation into ever more exotic taxa (reviewed in Elliot and Crespi, 
2009), combined with statistical models of the evolutionary process (Elliot and Crespi, 2009; Carter and Enders, 2004; Vogel, 2005; Elliot and Crespi, 2008; Wildman et al., 2006; Mess and Carter, 2006), suggest strongly that the opposite is the case. At the present time it seems likely that the earliest true placental mammals were characterized already by hemochorial or endotheliochorial placentas, and that the epitheliochorial form of placentation is a relatively recent evolutionary novelty that has arisen multiple times, independently, in several taxonomic groups. The human hemochorial placenta, than, is in some respects actually a "primitive" one, and its lineage can be traced back without break to the earliest mammalian species that lived 200 million years ago.

In this speculative paper, I assess the evidence that epitheliochorial placentation may provide a protective effect against preeclampsia. I pay specific attention to oxidative stress during placentation, a factor which appears to play a major role in the pathogenesis of preeclampsia. I argue - on the basis of life history - that human beings and other apes may have "the wrong type" of placenta when viewed in the context of mammals as a whole, and suggest that the invasive hemochorial human placenta is not an adaptive evolutionary strategy at all, but perhaps reflects nothing more than a "frozen accident", a suboptimal phenotype that is maintained not by evolutionary selective advantage but by constraints imposed upon placentation by the contingencies of developmental biology. My view is that the central evolutionary problem of human pregnancy is not, "why is the human placenta so invasive?" but rather "why is the human placenta not epitheliochorial?".

\section{Oxidative stress in placentation and preeclampsia}

Oxidative stress arises during pregnancy either from an excessive production by the placental metabolism of reactive oxygen species (ROS), or from an inadequate supply of antioxidant substances to dispose of or suppress formation of these harmful free radicals (Wisdom et al., 1991; Myatt and Cui, 2004; Burton and Jauniaux, 2004, 2011; Lázár, 2012). The majority of ROS are thought to result from mitochondrial activity under hyperoxia (in which case, there are more electrons traveling along the mitochondrial electron transport chain and hence a greater chance of "leakage" of electrons onto molecular oxygen to generate the reactive superoxide ion) or hypoxia (in which case a shortage of molecular oxygen results in accumulation of electrons). But ROS can also be formed in the endoplasmic reticulum and other 
membranes (especially in highly secretory cells), by the enzyme NADPH oxidase (which is an especially important source in early pregnancy; Raijmakers et al., 2006), and by other enzymatic systems. Reactive oxygen species cause cellular stress by directly damaging DNA, proteins and lipids, and through their effects on intracellular signaling pathways that can give rise a wide range of cellular responses including proliferation, growth arrest, senescence and cell death (Martindale and Holbrook, 2002).

Reactive oxygen species are important signal transducers in normal placentation. In early pregnancy, embryos require low oxygen tension to cause development, through pathways activated by hypoxia-inducible factor, of blood, vasculature, placentas and nervous systems (Burton et al., 2003; Simon and Keith, 2008). Low oxygen tension in early pregnancy is also an important promoter of placental angiogenesis (Pereira et al., 2015). At this time, the embryo is supported by secretions from endometrial glands rather than from the maternal circulation (Jauniaux et al., 2000; Burton et al., 2002), but breaching of maternal blood vessels in the first trimester results in direct access to oxygenated maternal blood and a precipitous oxidative insult (Jauniaux et al., 2000). Under low oxygen tension trophoblast maintains a proliferative phenotype but under high oxygen tension trophoblast differentiates along a pathway leading to a highly invasive phenotype that is important in carrying out the remodeling of the endometrium in normal pregnancy (Genbacev, 1997). The formation of ROS under high oxygen tension is thought to be involved in several of the mechanisms by which trophoblast cells sense the degree of local oxygenation and program cell behavior accordingly (De Marco and Caniggia, 2002) and is involved in causing the fusing of cytotrophoblast cells into the syncytiotrophoblast (Frendo et al., 2001). Mounting evidence suggests that placental oxidative stress, in general, increases throughout the duration of pregnancy (Toescu et al., 2002; Qanungo and Mukherjea, 2000) although the concentration of oxygen declines after the initial hyperoxia (Soothill et al., 1986; Burton, 2009).

Medical research has provided evidence of increased oxidative stress of placental tissues, particularly the invasive cytotrophoblast, in preeclampsia (Sikkema et al., 2001; Many et al., 2000). Furthermore, the degree of oxidative stress appears to be correlated with the severity of preeclampsia (Chamy et al., 2006; Uotila et al., 1993). It is thought that inadequate differentiation of cytotrophoblast down the invasive pathway yields insufficient or unsuccessful remodeling of spiral arteries (characteristic of some but not all cases of preeclampsia), resulting in various forms of oxidative stress in the ma- 
ture placenta (Roberts and Hubel, 1999). These effects are not thought to be a consequence merely of inadequate oxygenation. Instead it is proposed that a lack of constancy in the concentration of oxygen may be more significant in generating oxidative stress (Burton and Jauniaux, 2004) by causing ischemia-reperfusion injuries. Under this scenario, reactive oxygen species are generated rapidly during reoxygenation following a local episode of transient hypoxia (Hung et al., 2001), causing apoptosis of trophoblast cells and the release of cellular debris and other materials into the maternal circulatory system (Redman and Sargent, 2000; Hung et al., 2002; Hung and Burton, 2006) and generating maternal endothelial inflammation and damage. To the extent that the severity of preeclampsia may depend upon the mother's ability to resist harmful substances emitted by oxidatively stressed placentas, pregnancy has been regarded as a "stress test" for the vascular health of a mother's body Sattar and Greer (2002).

\section{Placental structure and oxidative stress}

I hypothesize that the epitheliochorial placenta provides a structure in which the maternal circulatory system is buffered from direct exposure to reactive oxygen species and other stressors emitted by the fetal trophoblast by maternal cell layers including epithelium, connective tissue and endothelium. Maternal blood vessels supplying the epitheliochorial placenta are typical of mammalian vasculature in general, in exhibiting a progressive reduction of vessel diameter as the vessel approaches its target cells (Brosens et al., 1967). The situation in the hemochorial placenta is quite different, with vessels becoming progressively larger and less tense, resulting in very low peripheral resistance at the point at which maternal blood is deposited directly into the trophoblastic lacunae (Moll et al., 1975; Moffett and Loke, 2006). The hemochorial placenta thus appears to be adapted for direct transfer of materials between fetal and maternal bloodstreams, giving rise to a risk of the transfer in the opposite direction of harmful substances, while the epitheliochorial placenta often depends on direct secretion of materials from mother to fetus, the separation of blood mitigating against maternal danger. For example, iron is taken up by fetuses with hemochorial placentation directly from maternal blood in the form of transferrin, but must be actively secreted by uterine glands in the form of an iron-rich glycoprotein, uteroferrin, in species with epitheliochorial placentation (Carter, 2012); similarly, while some substances, such as glucose, appear to be able to diffuse across the several cell 
layers separating maternal blood and fetal blood in epitheliochorial placentation, other substances are transferred in the form of "uterine milk", an evolutionary novelty in epitheliochorial species (Hansen et al., 1987; Carter and Enders, 2013), or via various adaptations toward histotrophic nutrition (Carter and Enders, 2013).

Empirical evidence bearing on the notion that placental type may modify maternal risk of exposure to fetally-derived stressors has been gathered only from only very few species with hemochorial and epitheliochorial placentation. Moll and Kastendieck (1978) point out that the epitheliochorial placenta is impermeable to lactic acid, a byproduct of hypoxia, such that the fetal metabolism alone is responsible for clearing lactate to normal levels after periods of hypoxia (see also Prior, 1980). In species with hemochorial placentas, on the other hand, lactate can be excreted directly into the mother's circulatory system, making use of her metabolism to clear lactic acid more rapidly. Hence, in the case of lactate clearance, hemochorial placentation appears to provide a benefit to the fetus at the expense of the mother. Jones et al. (2010) study the expression of antioxidants over the course of pregnancy in the junctional zone and labyrinth of the hemochorial rat placenta. In rat gestation, there is evidence of increased oxidative stress over the course of pregnancy, accommodated by increased placental expression of antioxidant defense genes over time. Expression of Sod1 and Ucp2 mRNAs (antioxidants) and the activity of xanthine oxidase (a source of reactive oxygen species), all increased from days 16 to 22 of pregnancy in the labyrinth and junctional zone; Sod2 and Gpx3 (antioxidants) increased only in the rapidly growing labyrinth. A similar study in epitheliochorial sheep placentomes (Garrel et al., 2010) found that the expression of SOD1 did not vary significantly over the course of pregnancy, while the expression of SOD2 actually decreased from days 35 to 55. These findings, if they can be tentatively generalized to the class of hemochorial and epitheliochorial placentas, suggest that hemochorial placentas may be in some sense "more dangerous" than epitheliochorial placentas at least with respect to the kinds of cellular stress attenuated by the superoxide dismutases, which are known to be underexpressed in pregnancy-induced hypertension and overexpressed in preeclampsia in human beings (Wilson et al., 1994; Sikkema et al., 2001) and which are the main type of reactive oxygen species produced during placentation (Myatt and Cui, 2004). Interestingly, the superoxide dismutases show evidence of being targets of natural selection during an evolutionary increase in placental invasiveness at the origin of the great apes (Crosley 
et al., 2013). However, this is not to say that epitheliochorial placentas do not produce reactive oxygen species at all, of course. A number of non-SOD antioxidants were found to increase over the course of pregnancy in sheep, including glutathione peroxidase and glutathione reductase (Garrel et al., 2010). Studies of the epitheliochorial placenta of camels indicate increased oxidative stress during pregnancy in comparison with non-pregnant uteri, as measured by increased malon dialdehyde (an end product of lipid peroxidation) and uric acid (an antioxidant), and in ruminants in general, however no data is available on secular trends in oxidative stress over time in these species (Aurousseau et al., 2006; Al-Zamely, 2011).

Many ROS are confined to the cellular compartments in which they are formed, because their charge prevents passage across lipid membranes. While fetally derived ROS are known to generate preeclampsia-like symptoms when input into the maternal circulation (Mishra et al., 2011; Gupta et al., 2005), an alternative source of maternal pollution takes the form of microparticles, syncytiotrophoblast fragments and other cellular debris that are shed directly into the maternal circulatory system by oxidatively stressed trophoblast (Redman and Sargent, 2000). In an in vitro study of the oxidatively stressed hemochorial placenta, ninety percent of such debris was shed directly into the maternal rather than fetal circulatory compartment (Di Santo et al., 2007), a situation that cannot arise in epitheliochorial species in which maternal blood has no direct contact with stressed placental tissues. While microparticles play a role in maternal-fetal crosstalk and placental development in healthy pregnancies (Aharon and Brenner, 2013), their misregulation is thought to also be involved in a number of pathological processes including systemic maternal inflammation and endothelial dysfunction in preeclampsia (van der Post et al., 2011; Goswami et al., 2006)

The degree of oxidative stress encountered by cells is, in part, dependent on the environmental partial pressure of oxygen in the placenta and other tissues (Aurousseau et al., 2006; Thom et al., 2002; Finne et al., 2008). It has been hypothesized that, in addition to stress resulting from hypoxia and hyperoxia (Sibley et al., 2002; Huppertz et al., 2014; van Patot et al., 2012), the phenomenon of ischemia-reperfusion, in which blood flow is temporarily stopped such that tissues become anoxic and are later damaged during reperfusion (Jones, 1985), also plays a role. Huppertz (2008) points out that inadequate and incomplete trophoblast invasion of maternal spiral arteries associated with hypoxia is a particular characteristic of early-onset preeclampsia (around 20\% of cases) rather than late-onset; disruptions in 
oxygen sensing in preeclampsia are likely of particular importance during early gestation (Rolfo et al., 2010). As described above, transient rapid changes in the partial pressure of oxygen are important components of signaling systems involved in early placental development, such as the adoption by trophoblast of an invasive phenotype following increased oxygen exposure after breaching of maternal vessels by fetal tissues in hemochorial placentation (Burton, 2009; Caniggia et al., 2000). The invasive phenotype exhibited by pig trophoblast when transplanted to an ectopic site (Samuel and Perry, 1972) suggests that trophoblast from species with epitheliochorial placentas have retained the ability to invade deeply but it is prevented by the persistence of maternal uterine epithelium throughout pregnancy, with the result that trophoblasts in epitheliochorial species are not exposed to the sudden burst of oxygen partial pressure necessary to promote formation of hemochorial placentas following implantation. Moll et al. (1975) point out that the hemochorial placenta (but not the epitheliochorial placenta) exhibits a state very unusual in higher vertebrates, and more characteristic of lower animals such as insects, in which maternal blood is not bound by vessels but is instead part of an open circulatory system at the maternal-fetal interface. Ultrasonography indicates that blood flow in the open circulatory system is homogeneous in early human pregnancy but later becomes heterogeneous, with areas of decreased echogenicity associated with "placental lakes" and increased echogenicity associated with "placental caverns", and the presence of intervillous thrombi (Kennedy et al., 2014). While the clinical significance of this variation, in normal pregnancies, is unclear (Jauniaux and Nicolaides, 1996; Hwang et al., 2012; Reis et al., 2005; Thompson et al., 2002), it seems likely that heterogeneity in placental perfusion arising from the open circulatory system will put species with hemochorial placentas at greater risk of ischemia-reperfusion-type injuries involving oxidative stress than species with epitheliochorial placentas, in which maternal blood circulation is more typical of mammals in being constrained to closed vessels. Variation in the degree of oxygenation of placental tissues also gives rise to spatial variation in the degree of oxidative stress over the placenta (Jauniaux et al., 2003). Empirical evidence suggests that the partial pressure of oxygen, over the course of pregnancy, is in general stable - exhibiting little change over the course of pregnancy, and resistant to experimental modification - in the epitheliochorial placentas of species such as sheep and goat (Comline and Silver, 1968; Meschia et al., 1965). 


\section{Life history and the evolution of epitheliochorial placentation}

I have argued that epitheliochorial placentation likely provides a protective effect against uterine cellular stress by providing a barrier to reactive oxygen species, microparticles, cellular debris and other stressors released by the oxidatively stressed placenta, and by reducing the heterogeneity over space and time of placental perfusion, thereby reducing direct stress on maternal cells and also reducing the risk of ischemia-reperfusion-type injuries that underly the pathophysiology of preeclampsia. This is consistent with the fact that preeclampsia is unknown in any species with epitheliochorial placentation. The majority of cases of preeclampsia occur in the third trimester, when the placenta reaches its greatest size, and is more likely in multiple pregnancies, in which the placenta grows larger (Redman and Sargent, 2000). Preeclampsia is also more common in women bearing large than small benign trophoblastic tumors (Llewellyn-Jones, 1967). Hence we may say that, on the whole, late onset preeclampsia is primarily a disease of large placentas. Since the size of a placenta is closely correlated with gestation time (Thomson et al., 1969), we might also regard late onset preeclampsia as primarily a disease of long gestations (note that the primary treatment for preeclampsia - delivery - amounts to an artificial reduction of human gestation length). In the context of oxidative stress, these interpretations seem reasonable. Total antioxidant capacity and titers of lipid hydroperoxides in maternal blood both increase gradually throughout normal pregnancy, indicating a general increase in oxidative stress as gestation proceeds and the placenta grows (Toescu et al., 2002; Qanungo and Mukherjea, 2000). As Burton and Jauniaux (2004) point out, "Qualitatively, the situation within the placenta in preeclampsia appears to be an extension of that seen towards the end of normal gestation, as there is evidence of increased nitrotyrosine formation, increased lipid peroxidation, increased trophoblast apoptosis, decreased activity of the principal antioxidant enzymes, and reduced tissue concentrations of nonenzymatic antioxidant molecules such as vitamin E" (emphasis mine).

We may predict, therefore, that epitheliochorial placentation is an advantageous adaptation for mammalian species that have evolved unusually extended gestation lengths, or unusually large offspring (with concomitantly large placentas) because it affords mothers some protection against the consequences of placental cellular stress. Intriguingly, epitheliochorial placentation is indeed found overwhelmingly in large bodied species with long gestation lengths (Carter and Enders, 2013). Elliot and Crespi (2008) showed that 
when mothers and their offspring undergo evolutionary change towards larger body size, transitions from hemochorial to less invasive non-hemochorial forms of placentation are significantly more likely to occur than in the absence of such changes in body size. Hence, large bodied animals (such as ruminants, horses, rhinoceros and tapirs, and whales) tend to exhibit epitheliochorial placentation. Capellini et al. (2011) show that increased placental invasiveness and especially fine interdigition between fetal and maternal blood streams are associated with an average 66 percent reduction in gestation length across mammals as a whole (but see somewhat contradictory findings from Garratt et al., 2013). Furthermore, many epitheliochorial species give birth to singletons or small litters and precocial young (Carter and Enders, 2013), both traits which tend to be associated with large offspring (and correspondingly large placentas).

These empirical findings are broadly in line with the view that epitheliochorial placentation has evolved as a protective mechanism for mammalian mothers in species whose reproductive mode - large offspring birthed in small litters after an extended gestation period - tends to promote high levels of placental cellular stress.

\section{The evolutionary origins of preeclampsia risk}

A corollary of the hypotheses presented above is that risk of preeclampsia and other gestational disorders associated with oxidative stress should be highest in species with large offspring, long gestation lengths and hemochorial placentation. Preeclampsia is observed primarily in human beings, but has occasionally and tentatively been reported in other apes including gorillas (Baird, 1981; Thornton and Onwude, 1992) and chimpanzees (Stout and Lemmon, 1969), and monkeys including the macaque (and perhaps others; Krugner-Higby et al., 2009).

Primates in general exhibit "slow" life histories (Jones, 2011) characterized by long lives, long gestation lengths, late ages at first reproduction and low fertility. This life history is more exaggerated amongst the apes and is especially pronounced in human beings, which are "slow" even in comparison with their closest relative, the chimpanzee (Walker et al., 2006). On the basis of the power law relating maternal body mass to gestation length in primates as a whole, human pregnancy last 37 days longer than expected - a greater excess than is found in both gorilla and chimpanzee, and exceeded only by the orangutan among primates studied - suggesting that natural selection has 
favoured extended gestation lengths within the human lineage (Dunsworth et al., 2012). Furthermore, human babies are unusually "big and fat" (Pawlwski, 1998), perhaps associated with their large and fatty brains (Cunnane and Crawford, 2003). These life history traits, combined with hemochorial placentation, are expected under the hypothesis presented above to result in an unusually high risk of maternal oxidative stress and vascular damage during pregnancy in human beings, even in comparison with other apes which is of course what we observe.

The question of evolutionary interest, then, is - given the enormous cost in lives and health that preeclampsia must have wrought over the generations - why have human beings not evolved non-hemochorial forms of placentation in the same way that other large, slow-living, big-brained mammals such as the whales and elephants did? The primates present a remarkable reversal of the pattern found in other mammals, with the smaller species (strepsirhine primates: lorises, galagos, bushbabies and lemurs) exhibiting epitheliochorial placentation and the larger species (haplorhine primates: tarsiers, monkeys and apes) exhibiting hemochorial placentation. In the case of the strepsirhines, it is possible that breaking the pattern is illusory, since early strepsirhine primates, and especially some recently extinct lemurs, reached large sizes and the diminutive size of most extant species may reflect non-random extinction associated with human colonization (Jungers et al., 2002). However the apes and especially human beings present a suite of life history and placental traits that is most unusual in a broader mammalian context.

The notion that a highly invasive hemochorial placenta is necessary for development of the unusually large brain of human beings has been proposed a number times (i.e., Eisenberg, 1983; Robillard et al., 2002; Cole et al., 2008). If so, then preeclampsia risk is the price paid by human beings for our mental faculties. However, a number of studies (Elliot and Crespi, 2008; Capellini et al., 2011; Martin, 2007; Carter and Pijnenborg, 2011) find no evidence that species with hemochorial placentas typically have larger brains than species with non-hemochorial placentas. The whales, with brains larger than our own, but epitheliochorial placentation, are a case in point. Hence, an adaptive explanation in terms of the costs of preeclampsia being outweighed by benefits of rapid prenatal brain development supposedly afforded by a hemochorial placenta can not be regarded as well supported at the present time.

Non-adaptive evolutionary explanations do not appear to have been explored in the literature. We here return our attention to the effect of high 
oxygen tension on the developing trophoblast, specifically its induction of an invasive phenotype. As noted above, a similar phenotype arises in the trophoblast from species normally epitheliochorial, when transplanted into an ectopic location. I propose that, in species with interstitial implantation this phenotype is inevitable in that the blastocyst must develop deeply embedded in the tissues of the uterus, in close contact with oxygenated maternal blood. Interstitial implantation may thus act to channel placental development toward a hemochorial outcome and, in the long term, prevent the evolution of epitheliochorial placentation in a mechanistic rather than adaptive manner. A recent study of the evolution of embryo implantation in mammals (McGowen et al., 2014) tabulates data for mammalian species in which the mode of implantation is known, and provides support for this view. The evolutionary reconstructions by McGowen et al. (2014) and Elliot and Crespi (2009) together imply that all evolutionary transformations from hemochorial to epitheliochorial placentation have occurred in lineages with superficial implantation. While there exist species with superficial implantation and hemochorial placentation (i.e., elephant shrews), species with superficial implantation and epitheliochorial placentation (i.e., pigs, sheep, lemurs), and species with interstitial implantation and hemochorial placentation (i.e., hedgehogs, humans, guinea pigs), there is, remarkably, no mammalian species with both interstitial implantation and epitheliochorial placentation. Interstitial hemochorial placentation may be an example of irreversible evolution (Bull and Charnov, 1985).

Future research into the evolutionary origins of preeclampsia must take into account both the adaptive and maladaptive implications of different placental structures, but may also usefully focus on the mechanistic constraints of placental development that may render potentially maladaptive traits fixed in the human population.

\section{Acknowledgments}

I thank Pierre-Yves Robillard for his kind hospitality, and participants in the International Workshops on Reproductive Immunology, Immunological Tolerance and Immunology of Preeclampsia, for their helpful comments and suggestions. I thank St. John's College, Cambridge for supporting my attendance at the workshop.

Aharon, A., Brenner, B., 2013. Placenta-derived microparticles. Thrombosis Research 131, S22-S24. 
Al-Zamely, H., 2011. Oxidant-antioxidant status and some biochemical parameters in pregnant and non pregnant Iraqi she camels. The Iraqi Journal of Veterinary Medicine 35 (2), 46-51.

Aurousseau, B., Gruffat, D., Durand, D., Nov 2006. Gestation linked radical oxygen species fluxes and vitamins and trace mineral deficiencies in the ruminant. Reproduction Nutrition Development 46 (6), 601-620.

Baird, J., 1981. Eclampsia in a lowland gorilla. American Journal of Obstetrics and Gynecology 141 (3), 345-346.

Brosens, I., Robertson, W., Dixon, H., 1967. The physiological response of the vessels of the placental bed to normal pregnancy. The Journal of Pathology and Bacteriology 93 (2), 569-579.

Bull, J., Charnov, E., 1985. On irreversible evolution. Evolution, 1149-1155.

Burton, G., Hempstock, J., Jauniaux, E., 2003. Proceedings of a Workshop on Embryonic and Fetal Nutrition. Vol. 10 of Havemeyer Foundation Series. Havemeyer Foundation, Ch. Endometrial secretions and development of the human fetoplacental unit during early pregnancy, pp. 20-22.

Burton, G., Jauniaux, E., Sep 2004. Placental oxidative stress: From miscarriage to preeclampsia. Journal of the Society for Gynecologic Investigation 11 (6), 342-352.

Burton, G., Jauniaux, E., 2011. Oxidative stress. Best Practice \& Research Clinical Obstetrics \& Gynaecology 35, 287-299.

Burton, G. J., Jul 2009. Oxygen, the janus gas; its effects on human placental development and function. Journal of Anatomy 215 (1), 27-35.

Burton, G. J., Watson, A. L., Hempstock, J., Skepper, J. N., Jauniaux, E., 2002. Uterine glands provide histiotrophic nutrition for the human fetus during the first trimester of pregnancy. The Journal of Clinical Endocrinology \& Metabolism 87 (6), 2954-2959.

Caniggia, I., Winter, J., Lye, S., Post, M., Mar 2000. Oxygen and placental development during the first trimester: Implications for the pathophysiology of pre-eclampsia. Placenta 21, S25-S30. 
Capellini, I., Venditti, C., Barton, R., 2011. Placentation and maternal investment in mammals. American Naturalist 177 (1), 86-98.

Carter, A., Enders, A., 2013. The evolution of epitheliochorial placentation. Annual Reviews of Animal Science 1, 443-467.

Carter, A. M., 2012. Evolution of placental function in mammals: The molecular basis of gas and nutrient transfer, hormone secretion, and immune responses. Physiological Reviews 92 (4), 1543-1576.

Carter, A. M., Enders, A. C., 2004. Comparative aspects of trophoblast development and placentation. Reproductive Biology and Endocrinology $2(1), 46$.

Carter, A. M., Pijnenborg, R., 2011. Evolution of invasive placentation with special reference to non-human primates. Best Practice \& Research Clinical Obstetrics \& Gynaecology 25 (3), 249-257.

Chamy, V., Lepe, J., Catalan, A., Retamal, D., Escobar, J., Mardrid, E., 2006. Oxidative stress is closely related to clinical severity of pre-eclampsia. Biological Research 39 (2).

Cole, L. A., Khanlian, S. A., Kohorn, E. I., 2008. Evolution of the human brain, chorionic gonadotropin and hemochorial implantation of the placenta: insights into origins of pregnancy failures, preeclampsia and choriocarcinoma. The Journal of Reproductive Medicine 53 (8), 549-557.

Comline, R. S., Silver, M., 1968. po2 levels in the placental circulation of the mare and ewe. Nature 217 (5123), 76-77.

Crosley, E. J., Elliot, M. G., Christians, J. K., Crespi, B. J., Feb 2013. Placental invasion, preeclampsia risk and adaptive molecular evolution at the origin of the great apes: evidence from genome-wide analyses. Placenta 34 (2), 127-132.

Cunnane, S. C., Crawford, M. A., 2003. Survival of the fattest: fat babies were the key to evolution of the large human brain. Comparative Biochemistry and Physiology Part A: Molecular \& Integrative Physiology 136 (1), $17-26$. 
De Marco, C., Caniggia, I., Apr 2002. Mechanisms of oxygen sensing in human trophoblast cells. Placenta 23, S58-S68.

Di Santo, S., Sager, R., Andres, A.-C., Guller, S., Schneider, H., Apr 2007. Dual in vitro perfusion of an isolated cotyledon as a model to study the implication of changes in the third trimester placenta on preeclampsia. Placenta 28, S23-S32.

Dunsworth, H. M., Warrener, A. G., Deacon, T., Ellison, P. T., Pontzer, H., Aug 2012. Metabolic hypothesis for human altriciality. Proceedings of the National Academy of Sciences 109 (38), 15212-15216.

Eisenberg, J., 1983. The Mammalian Radiations: An Analysis of Trends in Evolution, Adaptation and Behavior. University of Chicago Press.

Elliot, M., Crespi, B., Nov 2008. Placental invasiveness and brain-body allometry in eutherian mammals. Journal of Evolutionary Biology 21 (6), 1763-1778.

Elliot, M., Crespi, B., Nov 2009. Phylogenetic evidence for early hemochorial placentation in eutheria. Placenta 30 (11), 949-967.

Elliot, M. G., Crespi, B. J., Jan 2015. Genetic recapitulation of human preeclampsia risk during convergent evolution of reduced placental invasiveness in eutherian mammals. Philosophical Transactions of the Royal Society B: Biological Sciences 370 (1663), 20140069-20140069.

Finne, E., Olsvik, P., Berntssen, M., Hylland, K., Tollefsen, K., Sep 2008. The partial pressure of oxygen affects biomarkers of oxidative stress in cultured rainbow trout (oncorhynchus mykiss) hepatocytes. Toxicology in Vitro 22 (6), 1657-1661.

Frendo, J.-L., Thérond, P., Bird, T., Massin, N., Muller, F., Guibourdenche, J., Luton, D., Vidaud, M., Anderson, W. B., Evain-Brion, D., 2001. Overexpression of copper zinc superoxide dismutase impairs human trophoblast cell fusion and differentiation. Endocrinology 142 (8), 3638-3648.

Garratt, M., Gaillard, J.-M., Brooks, R. C., Lemaître, J.-F., 2013. Diversification of the eutherian placenta is associated with changes in the pace of life. Proceedings of the National Academy of Sciences 110 (19), 7760-7765. 
Garrel, C., Fowler, P. A., Al-Gubory, K. H., Jan 2010. Developmental changes in antioxidant enzymatic defences against oxidative stress in sheep placentomes. Journal of Endocrinology 205 (1), 107-116.

Genbacev, O., Sep 1997. Regulation of human placental development by oxygen tension. Science 277 (5332), 1669-1672.

Goswami, D., Tannetta, D., Magee, L., Fuchisawa, A., Redman, C., Sargent, I., Von Dadelszen, P., 2006. Excess syncytiotrophoblast microparticle shedding is a feature of early-onset pre-eclampsia, but not normotensive intrauterine growth restriction. Placenta 27 (1), 56-61.

Gupta, S., Agarwal, A., Sharma, R., 2005. The role of placental oxidative stress and lipid peroxidation in preeclampsia. Obstetrical \& Gynecological Survey 60 (12), 807-16.

Hansen, P., Ing, N., Moffatt, R., Baumbach, G., Saunders, T., Bazer, F., Roberts, R., 1987. Biochemical characterization and biosynthesis of the uterine milk proteins of the pregnant sheep uterus. Biology of Reproduction 36 (2), 405-418.

Hung, T.-H., Burton, G. J., Sep 2006. Hypoxia and reoxygenation: a possible mechanism for placental oxidative stress in preeclampsia. Taiwanese Journal of Obstetrics and Gynecology 45 (3), 189-200.

Hung, T.-H., Skepper, J., Charnock-Jones, D., Burton, G., Jun 2002. Hypoxia-reoxygenation: a potent inducer of apoptotic changes in the human placenta and possible etiological factor in preeclampsia. Circulation Research 90 (12), 1274-1281.

Hung, T.-H., Skepper, J. N., Burton, G. J., Sep 2001. In vitro ischemiareperfusion injury in term human placenta as a model for oxidative stress in pathological pregnancies. The American Journal of Pathology 159 (3), 1031-1043.

Huppertz, B., Apr 2008. Placental origins of preeclampsia: challenging the current hypothesis. Hypertension 51 (4), 970-975.

Huppertz, B., Weiss, G., Moser, G., Mar 2014. Trophoblast invasion and oxygenation of the placenta: measurements versus presumptions. J. Reprod. Immunol. 101-102, 74-79. 
Hwang, H. S., Sohn, I. S., Kwon, H. S., 2012. The clinical significance of large placental lakes. European Journal of Obstetrics \& Gynecology and Reproductive Biology 162 (2), 139-143.

Jauniaux, E., Hempstock, J., Greenwold, N., Burton, G. J., 2003. Trophoblastic oxidative stress in relation to temporal and regional differences in maternal placental blood flow in normal and abnormal early pregnancies. The American Journal of Pathology 162 (1), 115-125.

Jauniaux, E., Nicolaides, K. H., Feb 1996. Placental lakes, absent umbilical artery diastolic flow and poor fetal growth in early pregnancy. Ultrasound in Obstetrics and Gynecology 7 (2), 141-144.

Jauniaux, E., Watson, A. L., Hempstock, J., Bao, Y.-P., Skepper, J. N., Burton, G. J., 2000. Onset of maternal arterial blood flow and placental oxidative stress: a possible factor in human early pregnancy failure. The American Journal of Pathology 157 (6), 2111-2122.

Jones, D., 1985. Oxidative Stress. Academic Press, London, Ch. The role of oxygen concentration in oxidative stress: hypoxic and hyperoxic models, pp. 152-189.

Jones, J., Sep 2011. Primates and the evolution of long, slow life histories. Current Biology 21 (18), R708-R717.

Jones, M. L., Mark, P. J., Lewis, J. L., Mori, T. A., Keelan, J. A., Waddell, B. J., Apr 2010. Antioxidant defenses in the rat placenta in late gestation: Increased labyrinthine expression of superoxide dismutases, glutathione peroxidase 3, and uncoupling protein 2. Biology of Reproduction 83 (2), 254-260.

Jungers, W., Godfrey, L., Simons, E., Wunderlich, R., Richmond, B., Chatrath, P., 2002. Reconstructing Behavior in the Primate Fossil Record. Advances in Primatology. Springer, New York, Ch. Ecomorphology and behavior of giant extinct lemurs from Madagascar, pp. 371-411.

Kennedy, A., Woodward, P., Sohaey, R., Tubbs, W., 2014. Obstetric ultrasonography: the placenta. Abdominal Imaging 22 (6), 602-610.

Krugner-Higby, L., Luck, M., Hartley, D., Crispen, H. M., Lubach, G. R., Coe, C. L., Aug 2009. High-risk pregnancy in rhesus monkeys ( macaca 
mulatta ): a case of ectopic, abdominal pregnancy with birth of a live, term infant, and a case of gestational diabetes complicated by pre-eclampsia. Journal of Medical Primatology 38 (4), 252-256.

Lázár, L., 2012. Oxidative Stress and Diseases. InTech Open Science, Ch. The role of oxidative stress in female reproduction and pregnancy.

Llewellyn-Jones, D., 1967. Relation of pregnancy toxaemia to trophoblastic tumours. British Medical Journal 3, 720.

Lyall, F., 2005. Priming and remodelling of human placental bed spiral arteries during pregnancy-a review. Placenta 26, S31-S36.

Many, A., Hubel, C. A., Fisher, S. J., Roberts, J. M., Zhou, Y., Jan 2000. Invasive cytotrophoblasts manifest evidence of oxidative stress in preeclampsia. The American Journal of Pathology 156 (1), 321-331.

Martin, R. D., 2007. The evolution of human reproduction: a primatological perspective. American Journal of Physical Anthropology 134 (S45), 59-84.

Martindale, J. L., Holbrook, N. J., Jun 2002. Cellular response to oxidative stress: Signaling for suicide and survival. Journal of Cellular Physiology $192(1), 1-15$.

McGowen, M. R., Erez, O., Romero, R., Wildman, D. E., 2014. The evolution of embryo implantation. International Journal of Developmental Biology 58 (2-3-4), 155-161.

Meschia, G., Cotter, J., Breathnach, C., Barron, D., 1965. The hemoglobin, oxygen, carbon dioxide and hydrogen ion concentrations in the umbilical bloods of sheep and goats as sampled via indwelling pastic catheters. Quarterly Journal of Experimental Physiology and Cognate Medical Sciences 50, 185-195.

Mess, A., Carter, A. M., 2006. Evolutionary transformations of fetal membrane characters in eutheria with special reference to afrotheria. Journal of Experimental Zoology Part B: Molecular and Developmental Evolution 306 (2), 140-163.

Mishra, N., Nugent, W. H., Mahavadi, S., Walsh, S. W., Sep 2011. Mechanisms of enhanced vascular reactivity in preeclampsia. Hypertension 58 (5), 867-873. 
Moffett, A., Loke, C., Aug 2006. Immunology of placentation in eutherian mammals. Nature Reviews Immunology 6 (8), 584-594.

Moll, W., Kastendieck, E., 1978. Accumulation and disappearance of lactate in a fetus with a hemochorial placenta. the role of placental transfer and fetal metabolism. Journal of Perinatal Medicine 6, 246-254.

Moll, W., Künzel, W., Herberger, J., 1975. Hemodynamic implications of hemochorial placentation. European Journal of Obstetrics, Gynecology, and Reproductive Biology 5 (1-2), 67-74.

Myatt, L., Cui, X., 2004. Oxidative stress in the placenta. Histochemistry and Cell Biology 122 (4), 369-382.

Pawlwski, B., 1998. Why are human newborns so big and fat? Human Evolution 13 (1), 65-72.

Pereira, R. D., De Long, N. E., Wang, R. C., Yazdi, F. T., Holloway, A. C., Raha, S., 2015. Angiogenesis in the placenta: The role of reactive oxygen species signaling. BioMed Research International 2015, 1-12.

Pijnenborg, R., Vercruysse, L., Hanssens, M., Jan 2008. Fetal-maternal conflict, trophoblast invasion, preeclampsia, and the Red Queen. Hypertension in Pregnancy 27 (2), 183-196.

Prior, R., 1980. Glucose and lactate metabolism in vivo in ovine fetus. American Journal of Physiology 239 (3), E208-E214.

Qanungo, S., Mukherjea, M., 2000. Ontogenetic profile of some antioxidants and lipid peroxidation in human placental and fetal tissues. Molecular and Cellular Biochemistry 215, 11-19.

Raijmakers, M., Burton, G., Jauniaux, E., Seed, P., Peters, W., Steegers, E., Poston, L., 2006. Placental nad(p)h oxidase mediated superoxide generation in early pregnancy. Placenta 27 (2), 158-163.

Redman, C., Sargent, I., Sep 2000. Placental debris, oxidative stress and pre-eclampsia. Placenta 21 (7), 597-602. 
Reis, N. S. V., Brizot, M. L., Schultz, R., Nomura, R. M. Y., Zugaib, M., 2005. Placental lakes on sonographic examination: Correlation with obstetric outcome and pathologic findings. Journal of Clinical Ultrasound $33(2), 67-71$.

Roberts, J. M., Hubel, C. A., 1999. Is oxidative stress the link in the twostage model of pre-eclampsia? The Lancet 354 (91-81), 788-789.

Robillard, P.-Y., Dekker, G., Hulsey, T., Feb 2002. Evolutionary adaptations to pre-eclampsia/eclampsia in humans: Low fecundability rate, loss of oestrus, prohibitions of incest and systematic polyandry. American Journal of Reproductive Immunology 47 (2), 104-111.

Robillard, P.-Y., Hulsey, T. C., Dekker, G. A., Chaouat, G., 2003. Preeclampsia and human reproduction.: An essay of a long term reflection. Journal of Reproductive Immunology 59 (2), 93-100.

Rolfo, A., Many, A., Racano, A., Tal, R., Tagliaferro, A., Letta, F., Wang, J., Post, M., Caniggia, I., 2010. PLoS One 5 (10), e13288.

Rosenberg, K. R., Trevathan, W. R., Dec 2007. An anthropological perspective on the evolutionary context of preeclampsia in humans. Journal of Reproductive Immunology 76 (1-2), 91-97.

Samuel, C., Perry, J., 1972. The ultrastructure of pig trophoblast transplanted to an ectopic site in the uterine wall. Journal of Anatomy 113 (1), 139-149.

Sattar, N., Greer, I., Jul 2002. Pregnancy complications and maternal cardiovascular risk: opportunities for intervention and screening? British Medical Journal 325 (7356), 157-160.

Sibley, C. P., Pardi, G., Cetin, I., Todros, T., Piccoli, E., Kaufmann, P., Huppertz, B., Bulfamante, G., Cribiu, F. M., Ayuk, P., Glazier, J., Radaelli, T., Apr 2002. Pathogenesis of intrauterine growth restriction (IUGR)conclusions derived from a European Union Biomed 2 Concerted Action project 'Importance of Oxygen Supply in Intrauterine Growth Restricted Pregnancies' - a workshop report. Placenta 23 Suppl A, S75-79. 
Sikkema, J., van Rijn, B., Franx, A., Bruinse, H., de Roos, R., Stroes, E., van Faassen, E., Apr 2001. Placental superoxide is increased in pre-eclampsia. Placenta 22 (4), 304-308.

Simon, M. C., Keith, B., Feb 2008. The role of oxygen availability in embryonic development and stem cell function. Nature Reviews Molecular Cell Biology 9 (4), 285-296.

Soothill, P. W., Nicolaides, K. H., Rodeck, C. H., Campbell, S., 1986. Effect of gestational age on fetal and intervillous blood gas and acid-base values in human pregnancy. Fetal Diagnosis and Therapy 1 (4), 168-175.

Stout, C., Lemmon, W. B., 1969. Glomerular capillary endothelial swelling in a pregnant chimpanzee. American Journal of Obstetrics and Gynecology 105 (2), 212-215.

Thom, S. R., Bhopale, V., Fisher, D., Manevich, Y., Huang, P. L., Buerk, D. G., Mar 2002. Stimulation of nitric oxide synthase in cerebral cortex due to elevated partial pressures of oxygen: An oxidative stress response. Journal of Neurobiology 51 (2), 85-100.

Thompson, M., Vines, S., Aquilina, J., Wathen, N., Harrington, K., 2002. Are placental lakes of any clinical significance? Placenta 23 (8), 685-690.

Thomson, A. M., Billewicz, W. Z., Hytten, F. E., Oct 1969. The weight of the placenta in relation to birthweight. BJOG: An International Journal of Obstetrics \& Gynecology 76 (10), 865-872.

Thornton, J. G., Onwude, J. L., Jul 1992. Convulsions in pregnancy in related gorillas. American Journal of Obstetrics and Gynecology 167 (1), 240-241.

Toescu, V., Nuttall, S. L., Martin, U., Kendall, M. J., Dunne, F., Oct 2002. Oxidative stress and normal pregnancy. Clinical Endocrinology 57 (5), 609613.

Uotila, J., Tuimala, R., Aarnio, T., Pyykkö, K., Ahotupa, M., 1993. Findings on lipid peroxidation and antioxidant function in hypertensive complications of pregnancy. BJOG: An International Journal of Obstetrics \& Gynecology 100, 200-176. 
van der Post, J., Lok, C., Boer, K., Sturk, A., Sargent, I., Nieuwland, R., 2011. The functions of microparticles in pre-eclampsia. Seminars in Thrombosis and Hemostasis 37 (02), 146-152.

van Patot, M. C., Ebensperger, G., Gassmann, M., Llanos, A. J., Sep 2012. The hypoxic placenta. High Alt. Med. Biol. 13 (3), 176-184.

Vogel, P., 2005. The current molecular phylogeny of eutherian mammals challenges previous interpretations of placental evolution. Placenta 26 (8), 591-596.

Walker, R., Hill, K., Burger, O., Hurtado, M., 2006. Life in the slow lane revisited: ontogenetic separation between chimpanzees and humans. American Journal of Physical Anthropology 129, 577-583.

Wildman, D. E., Chen, C., Erez, O., Grossman, L. I., Goodman, M., Romero, R., 2006. Evolution of the mammalian placenta revealed by phylogenetic analysis. Proceedings of the National Academy of Sciences of the United States of America 103 (9), 3203-3206.

Wilson, R., Boyd, P., McKillop, J. H., Leitch, C., Walker, J. J., Burdon, R. H., Chen, G., Jan 1994. Normal superoxide dismutase (sod) gene in pregnancy-induced hypertension: Is the decreased sod activity a secondary phenomenon? Free Radical Research 21 (2), 59-66.

Wisdom, S., Wilson, R., McKillop, J., Walker, J., 1991. Antioxidant systems in normal pregnancy and in pregnancy-induced hypertension. American Journal of Obstetrics and Gynecology 165, 1701-1704. 